\title{
OPEN Integrated physiological, proteomic, and metabolomic analyses of pecan cultivar 'Pawnee' adaptation to salt stress
}

\begin{abstract}
Yun Jiao ${ }^{1}$, Jianhong Zhang ${ }^{1,2 \bowtie} \&$ Cunde Pan $^{2}$
The pecan is a salt-alkali-tolerant plant, and its fruit and wood have high economic value. This study aimed to explore the molecular mechanisms responsible for salt stress tolerance in the pecan grown under hydroponic conditions to simulate salt stress. The results showed that the photosynthetic rate $(P n)$ was reduced in response to salt stress, while the intercellular carbon dioxide concentrations $(\mathrm{Ci})$ increased. The response of the pecan to salt stress was measured using iTRAQ (isobaric tags for relative or absolute quantitation) and LC/MS (liquid chromatography and mass spectrometry) non-targeted metabolomics technology. A total of 198 differentially expressed proteins (65 downregulated and 133 up-regulated) and 538 differentially expressed metabolites ( 283 down-regulated and 255 up-regulated) were identified after exposure to salt stress for $48 \mathrm{~h}$. These genes were associated with 21 core pathways, shown by Kyoto Encyclopedia of Genes and Genomes annotation and enrichment, including the metabolic pathways involved in nucleotide sugar and amino sugar metabolism, amino acid biosynthesis, starch and sucrose metabolism, and phenylpropane biosynthesis. In addition, analysis of interactions between the differentially expressed proteins and metabolites showed that two key nodes of the salt stress regulatory network, L-fucose and succinate, were up-regulated and down-regulated, respectively, suggesting that these metabolites may be significant for adaptations to salt stress. Finally, several key proteins were further verified by parallel reaction monitoring. In conclusion, this study used physiological, proteomic, and metabolomic methods to provide an important preliminary foundation for improving the salt tolerance of pecans.
\end{abstract}

Leaves are the primary sites of photosynthesis and energy exchange in plants, and are highly sensitive to environmental conditions $s^{1,2}$. Salt stress can affect plant growth by reducing photosynthesis, with more severe effects seen with higher salt concentrations and longer exposure times directly or indirectly, resulting in reduced photosynthesis ${ }^{3}$. Transcriptomic analysis of pecan leaves in the early stages of salt stress has identified a large number of genes that respond to salt stress ${ }^{4}$. However, simple transcriptomic analysis cannot provide a comprehensive analysis of the molecular mechanism of salt stress adaptation, which requires in-depth investigation based on proteomics and metabolomics.

Quantitative proteomic methods, such as isobaric tag for relative and absolute quantitation (iTRAQ) and metabolomic techniques, such as liquid chromatography and mass spectrometry (LC/MS) allow in-depth analyses of plant metabolic adaptations to salt stress. These platforms have been used to integrate and analyze changes in barley (Hordeum spontaneum L. ${ }^{5}$, Arabidopsis thaliana ${ }^{6}$, wheat ${ }^{7}$, Malus halliana $^{8}$, rice $^{9}$, maize ${ }^{10}$, small rust fungus ${ }^{11}$, and sugar beet ${ }^{12}$ under salt stress conditions. For instance, significant changes in the contents of soluble sugars were found after wheat seedlings were inoculated with the wp- 6 strain (plant growth-promoting rhizosphere bacteria) under salt stress; these included proteins related to energy production and conversion and the metabolites palmitoleic acid, chlorophyll b, D-arginine, pheophytin a, rutin, and vanillin, suggesting that these may play important roles in promoting the growth of salt-stressed wheat seedlings ${ }^{7}$. Similarly, the salt-tolerant sugar beet T510 variety showed increased growth, together with higher sucrose and proline levels, and increased antioxidant enzyme activity in response to salt stress. The differentially expressed proteins (DEPs) identified by iTRAQ in sugar beet were found to be involved in many different activities, including photosynthesis, stress

\footnotetext{
${ }^{1}$ Institute of Forestry, Ningbo Academy of Agricultural Science, No. 19 Dehou Street, Ningbo 315040, Zhejiang, People's Republic of China. ${ }^{2}$ College of Forestry and Horticulture, Xinjiang Agricultural University, No. 311 Nongda East Road, Ürümqi 830052, Xingjiang, People's Republic of China. ${ }^{\varpi}$ email: nbjianhong@163.com
} 
and defense, protein synthesis, and signal transduction ${ }^{12}$. It is apparent that plants accumulate sugar, proline, secondary metabolites, and solutes related to osmotic adjustment on exposure to salt stress, as well as developing adaptive strategies and increasing their antioxidant capabilities, which the intrinsic mechanism of plant in response to salt stress has been recognized by many researchers. Interestingly, sugar not only participates in osmotic regulation, but also acts as a signaling molecule, sensing environmental stress and activating defense responses through the regulation of gene and protein expression and metabolite accumulation. For instance, sucrose promotes the accumulation of tryptophan, certain alkaloids, and D-phenylalanine, and can also activate glutamate-related proteins (ASN1, ASP3, GLN1-1, and NIT4) and aspartic acid, leading to the control of reactive oxygen species (ROS) levels and the induction of flavonoid biosynthesis and auxin signaling ${ }^{8}$. These reactions enhance the resistance of $M$. halliana to salt-alkali stress. Furthermore, glucose and fructose, acting as signaling molecules, have been shown to activate downstream salt-stress responses by regulating the expression of specific proteins ${ }^{13,14}$.

In addition, some proteins, such as heat shock proteins (HSPs) ${ }^{15}$, mitogen-activated protein kinases $(\mathrm{MAPKs})^{16}$, and the photosynthetic protein $\mathrm{PSBO} 2^{17}$, are thought to be involved in a variety of signal transduction pathways related to the salt-stress response, thereby increasing resistance to abiotic stress. For instance, MAPKs play crucial roles in the regulation of transcription factor gene expression under salt stress in rice $e^{18}$. Hsp17.6CII (a chaperone protein) in Arabidopsis activates CAT2 activity by regulating ROS levels, thus increasing the plant's resistance to abiotic stress ${ }^{19}$. Furthermore, several small molecules, including melatonin ${ }^{20}$ and $\gamma$-Aminobutyric acid (GABA) ${ }^{21}$, can enhance resistance to salt stress by triggering the expression of downstream proteins involved in the salt-stress response. Thus, the ability of halophytes to tolerate high salt levels is determined by a coordinated network of proteins and metabolites.

Current research on the molecular mechanisms of salt stress has concentrated mostly on model plants, and there are few studies on perennial woody oil plants. In this study, hydroponics was used to simulate the saltstress environment to comprehensively analyze the effects of salt stress on pecans. The physiological parameters relating to photosynthesis in the pecan leaves were analyzed, and used iTRAQ proteomics and non-targeted LC/ MS metabolomics to investigate the molecular regulatory mechanisms of pecans in response to salt stress with the goal of providing a theoretical basis for the future establishment of pecan cultivation in saline-alkali soils.

\section{Experimental details}

Plant material, salt-stress treatment, and analysis of photosynthetic parameters. Pecans were sourced and processed as previously described ${ }^{4}$. Specifically, samples of fully expanded leaves were collected from the plants and were then divided into three groups, with each group containing three replicates of six leaves. The groups were subjected to different $\mathrm{NaCl}$ concentrations and incubation times, as follows: group A, control group, no $\mathrm{NaCl}$ treatment, $0 \mathrm{~h}$; group $\mathrm{B}, 0.6 \% \mathrm{NaCl}$ for $24 \mathrm{~h}$; group $\mathrm{C}, 0.6 \% \mathrm{NaCl}$ for $48 \mathrm{~h}$. The samples were then rapidly frozen in liquid nitrogen for subsequent analysis. These analyses included the measurement of the photosynthetic rate $(\mathrm{Pn})$, stomatal conductance $(G s)$, and intercellular $\mathrm{CO}_{2}$ concentration $(\mathrm{Ci})$, measured on an LI-6400XT portable photosynthetic rate meter (Li-COR Biosciences, Lincoln, USA), on functional leaves at 10:00-11:00 (under natural light) on six leaves with three replicates per group (as below). At the same time, maximal fluorescence (Fm), the initial fluorescence (F0), maximal photochemical efficiency (Fv/Fm), light-induced nonphotochemical quenching (NPQ), nonregulatory energy dissipation (Y(NO)), and photochemical quenching coefficient $(\mathrm{qN})$ were measured in the leaves after incubation for $30 \mathrm{~min}$ in the dark using a chlorophyll fluorometer (PAM2500, Heinz Walz GmbH, Germany) and Pam-Win3 software. These values were exported to Excel (Microsoft Excel 2016) and analyzed using one-way ANOVA with $p$ values $<0.05$ considered statistically significant, using the Data Processing System software (version 14.10, Zhejiang University, Hangzhou, China). Finally, Adobe Illustrator 2019 (Adobe Systems, San Jose, CA, USA) and OriginPro software version 2021 (Northampton, MA, USA) were used to draw and edit the data maps.

Isobaric tags for relative and absolute quantitation (iTRAQ) analysis. Protein extracts from three biological replicates from each treatment group were used for digestion and ITRAQ labeling, as previously described ${ }^{12}$. The protein concentration was determined by the bicinchoninic acid (BCA) method ${ }^{22}$. The iTRAQ labeling was carried out according to the filter aided sample preparation (FASP) $\operatorname{method}^{23}$, and the components separated using an Agilent 1100 HPLC (Agilent Technologies, Palo Alto, CA, USA). The relevant parameters were as follows: flow rate, $300 \mu \mathrm{l} / \mathrm{min}$; mobile phase A phase, $\mathrm{H}_{2} \mathrm{O}-\mathrm{FA}$ (99.9:0.1, v /v); mobile phase $\mathrm{B}$ phase, $\mathrm{ACN}-\mathrm{H}_{2} \mathrm{O}-\mathrm{FA}$ (80:19.9:0.1, v/v/v); gradient elution conditions: 0-1 min, 2-9\% B; 1-45 min, 9-29\% B; 45-52 $\mathrm{min}, 29-37 \% \mathrm{~B}$; $52-56 \mathrm{~min}, 37-100 \% \mathrm{~B}$; 56-60 $\mathrm{min}, 100 \% \mathrm{~B}$. The mass spectrometry conditions were described in the reference ${ }^{24}$. Finally, Proteome Discoverer 2.4 software (Thermo Fisher Scientific, Bremen, Germany) was used to complete the protein identification and analysis. The mass spectrometry proteomics data have been uploaded to the Proteome Xchange Consortium (http://proteomecentral.proteomexchange.org) with the dataset identifier PXD030031 and the subject ID IPX0003700000.

LC/MS untargeted metabolomics analysis. Eight milligrams from each sample were weighed out, and $20 \mu \mathrm{l}$ internal standard ${ }^{25}$, and $1 \mathrm{ml}$ methanol: water $(\mathrm{V}: \mathrm{V}=7: 3)$ were added, and ground in a grinder $(60 \mathrm{~Hz}$, $2 \mathrm{~min}$ ). Ultrasonic extraction was performed for $30 \mathrm{~min}$, after which the sample was allowed to stand at $-20^{\circ} \mathrm{C}$ for $20 \mathrm{~min}$ and was then centrifuged for $10 \mathrm{~min}\left(13,000 \mathrm{rpm}, 4^{\circ} \mathrm{C}\right)$. Three hundred microliters of the supernatant were then evaporated to dryness, reconstituted with $400 \mu \mathrm{l}$ methanol-water $(\mathrm{V}: \mathrm{V}=1: 4)$, ultrasonicated for $2 \mathrm{~min}$, and centrifuged for $10 \mathrm{~min}\left(13,000 \mathrm{rpm}, 4^{\circ} \mathrm{C}\right)$, before withdrawing $150 \mu \mathrm{l}$ of the supernatant with a syringe. This was then filtered through a $0.22 \mu \mathrm{m}$ organic phase pinhole filter. LC/MS analysis was performed using a liquid mass spectrometry system composed of Dionex U3000 UHPLC and QE plus (Thermo Fisher, 
Waltham, MA, USA). Data collection and the identification of compounds were described in the reference ${ }^{26}$. Metabolic changes in the samples were investigated using principal component analysis (PCA) and (orthogonal) partial least-square discriminant analysis (O) PLS-DA. The variable importance in projection (VIP) was used to rank the overall contribution of each variable to the OPLS-DA model, and those variables with $p$ values $<0.05$ and VIP $>1$ were considered to be related to group discrimination. In addition, the Kyoto Encyclopedia of Genes and Genomes (KEGG, http://www.genome.jp/KEGG/pathway.html) was searched for metabolic pathway enrichment.

Data analysis and verification of iTRAQ data by PRM. To analyze the functional characteristics of the selected DEPs, annotation information was extracted from the gene ontology (GO) and KEGG databases using the search term Juglans regia "English walnut" ${ }^{27}$ for protein functional annotation and pathway enrichment analysis. In addition, the online analysis platform Paintomics 3 (http://www.paintomics.org/) was used for the correlation analysis between DEPs and metabolites, as well as the OmicShare online analysis cloud platform 6.4.5 (https://www.omicshare.com/tools/) to establish a network diagram of DEPs and metabolites. Among them, the core DEPs and metabolites were determined through the MetaboAnalyst 5.0 online analysis platform (https://www.metaboanalyst.ca/MetaboAnalyst/) to establish a regulatory network diagram. Furthermore, 20 proteins were chosen and quantified to verify the iTRAQ data by PRM analysis, with the detailed parameters for the liquid chromatography (LC) system and mass spectrometer taken from the relevant literature ${ }^{28}$. Three biological replicates of each group were performed. The PRM analysis is supported by Novogene Bioinformatics Institute (Beijing, China).

Ethics approval. The experimental research and field studies on plants, including the collection of plant material, complied with relevant institutional, national, and international guidelines and legislation. The appropriate permissions and/or licenses for the collection of plant or seed specimens were obtained for the study.

\section{Results}

Pecan leaves in response to salt stress and changes in photosynthesis. To understand the physiological response of pecans to salt stress, we treated pecans with $\mathrm{NaCl}$. After $24 \mathrm{~h}$, the morphological characteristics of the pecan leaves were not significantly different from those of the control (CK). Only after $48 \mathrm{~h}$ could a slight lightening of the color on the leaf edges be seen (Fig. S1). In addition, the photosynthetic rate $(\mathrm{Pn})$ showed a downward trend after $24 \mathrm{~h}$ treatment but the overall change was not large (Fig. 1). The stomatal conductance (Gs) showed an obvious upward trend after $24 \mathrm{~h}$ of salt-stress treatment. The intercellular carbon dioxide concentration $(\mathrm{Ci})$ of the leaves rose continuously with prolonged exposure to salt stress, implying that pecans may be mainly affected by stomal regulation. At the same time, the F0 and $\mathrm{Y}(\mathrm{NO})$ of the leaf fluorescence parameters showed an upward trend, indicating that the leaf tissue did not completely utilize the light energy absorbed by the plant, and that salt stress had already caused damage to the photosynthetic system. The decrease in Fv/Fm suggested a gradual increase in the photoinhibition response while NPQ and qN showed significant decreases after treatment for $24 \mathrm{~h}$, indicating a gradual reduction in the photoprotective ability of the leaf tissue and an inability to convert excess light energy into heat loss. Thus, although the leaves did not show marked reactions to salt stress treatment, photosynthesis was inhibited to a certain extent.

Proteomic analyses of pecan leaves in response to salt stress. Pecan leaves were evaluated using iTRAQ quantitative proteomics, and the adjusted $p$ values $<0.05$ were used as thresholds to screen for significant changes in DEP abundance. We identified 198 DEPs in the leaves; of these, 65 were down-regulated and 133 were up-regulated (Table S1). GO term enrichment analysis showed that the 198 DEPs were divided into three categories, including biological processes (BP), cellular components (CC), and molecular functions (MF) (Fig. 2A). The most abundant terms included fruit development, chitin catabolism, and photosystem II oxygen-evolving complex components in the BP category. The CC category included significant enrichment of membrane components, anchoring components of the plasma membrane, and the chloroplast thylakoid membrane. For the MF category, L-ascorbic acid-binding and polysaccharide-binding were the most abundant categories. The top 20 KEGG pathways are shown in Fig. 2B. The DEPs were mainly involved in the metabolic pathways phenylpropane biosynthesis, protein processing in the endoplasmic reticulum, photosynthesis, MAPK signaling pathway, amino sugar and nucleotide sugar metabolism, glutathione metabolism, and pentose and glucose conversion of aldehyde esters. These results indicate that proteins involved in the synthesis of metabolites respond to salt stress by regulating various metabolic pathways.

Metabolic analyses of pecan leaves in response to salt stress. Through LC-MS identification and the OPLS-DA model with $p$ value $<0.05$ and VIP $>1,538$ differentially expressed metabolites (DEMs) were identified. Of these, 255 were up-regulated and 283 were down-regulated (Table S2). These metabolites mainly included amino acids, sugars, organic acids, and secondary metabolites. The top 50 DEMs were analyzed through hierarchical clustering; the heatmap is shown in Fig. 3. All the DEMs affected by salt stress were investigated in the KEGG database to identify the pathways in which they were involved. The top 20 pathways included piperidine and pyridine alkaloid biosynthesis, tropane, $\alpha$-linolenic acid metabolism, galactose metabolism, ABC transporter, glycerophospholipid metabolism, lysine degradation, amino acid biosynthesis, 2-oxocarboxylic acid metabolism, and degradation of the limonene and pinene (Fig. 4). The changes in these metabolic pathways and metabolites provide important information to explain the response mechanism of pecans to salt stress. 

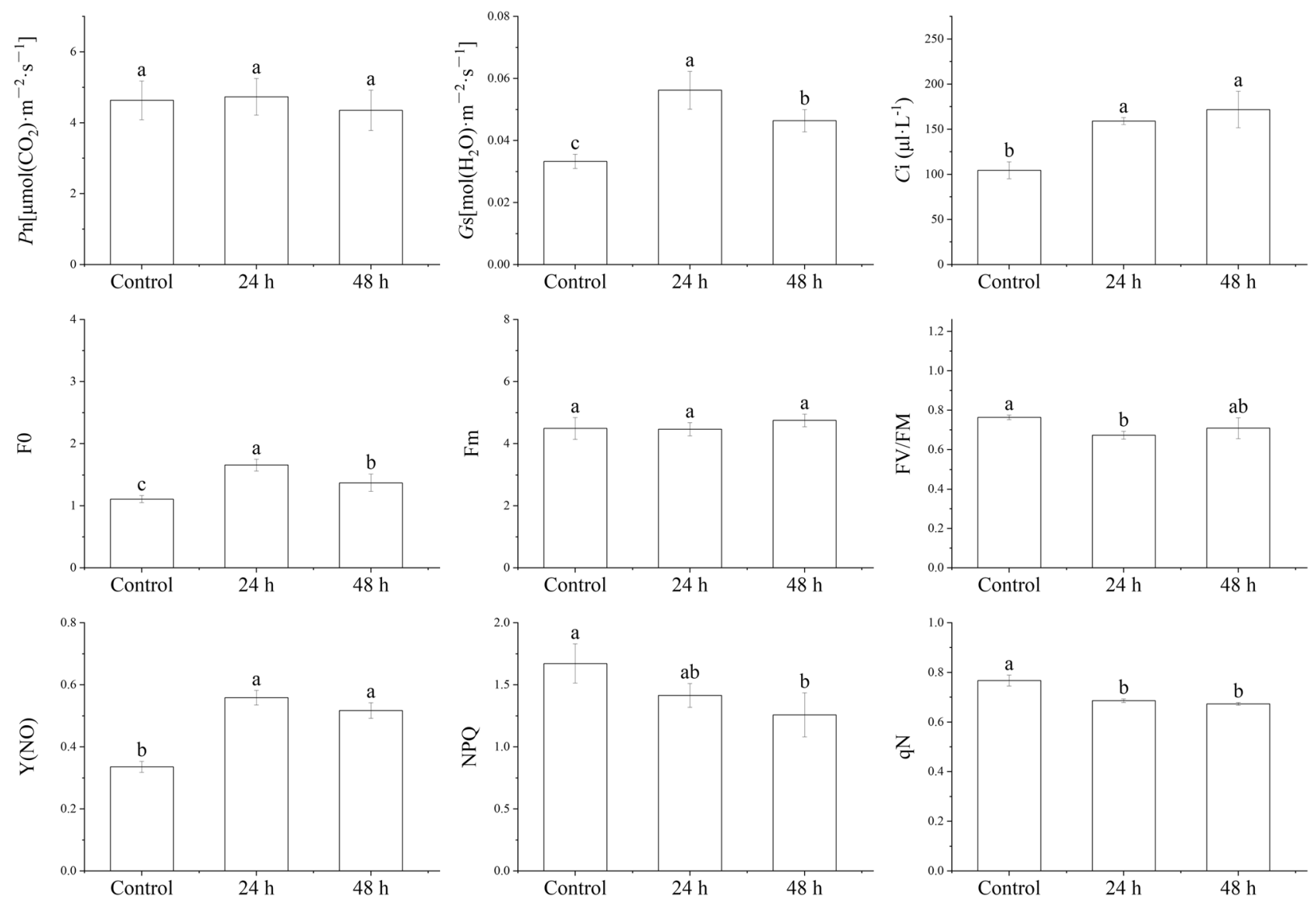

Figure 1. Physiological parameters of pecan under salt stress: photosynthetic rate $(P \mathrm{n})$; stomatal conductance $(\mathrm{Gs})$; intercellular $\mathrm{CO}_{2}$ concentration $(\mathrm{Ci})$; initial fluorescence (F0); maximal fluorescence (Fm); maximal photochemical efficiency (Fv/Fm); light-induced nonphotochemical quenching (NPQ); nonregulatory energy dissipation ( $\mathrm{Y}(\mathrm{NO})$ ); photochemical quenching coefficient (qN).

Correlation analysis of proteomics and metabolomics of leaves in response to salt stress. Analysis of the correlation between proteins and metabolites under salt stress showed that 23 metabolic pathways were enriched (Fig. 5); these included 93 proteins that were assigned to these pathways. These metabolic pathways included nucleotide sugar and amino sugar metabolism, starch and sucrose metabolism, amino acid biosynthesis, and phenylpropane biosynthesis. A regulatory network diagram of the core DEPs and DEMs included 21 metabolic pathways, indicating the response of the pecan leaves to salt stress (Fig. 6, Table 1). It can be clearly seen that there was a continuous accumulation of L-fucose, sucrose, L-arabinose, and beta-D-glucosyl2 -coumarinate in response to salt stress. Of these, L-arabinose $\left(\log _{2}\right.$ Fold change $\left.=3.96\right)$ and beta-D-glucosyl2 -coumarinate $\left(\log _{2}\right.$ Fold change $\left.=2.70\right)$ showed significantly raised levels, and together with L-fucose, were the key nodes in the salt stress regulatory network. In contrast, succinate was down-regulated. Since the succinate participates in multiple metabolic regulation pathways and is a downstream component of metabolic regulation, indicating that metabolic pathways involving succinate may be key to the response to salt stress. In addition, the down-regulation of LOC109005850 (AT1G31690) expression involving the tropane, piperidine, and pyridine alkaloid biosynthetic pathway suggested that it could be involved in the reduced accumulation of four metabolites, namely, 5-aminopentanal, ecgonine methyl ester, niacinamide, and tropine. However, further verification is required.

Verification of DEP analysis results by PRM. In order to evaluate the validity of the experimental results by iTRAQ analysis, we selected 20 proteins (LOC108981203, LOC108986524, LOC108986997, LOC108987294, LOC108990523, LOC108993800, LOC108995000, LOC108995788, LOC108998475, LOC109003002, LOC109005150, LOC109005909, LOC109007845, LOC109010267, LOC109010467, LOC109010561, LOC109011398, LOC109011575, LOC109012612, LOC109020284) from various pathways, including those of fructose metabolism, glutamine biosynthesis, glucose metabolism, photosynthetic subunit of subcomplex, ribosomal protein, and HSPs, and performed PRM analysis (Table 2, Table S3). The results indicated that 19 proteins (95\%) showed similar trends between the iTRAQ analysis and PRM results, indicating the reliability of the iTRAQ analysis, but LOC109005150 analyzed by PRM was not consistent with iTRAQ data. 
a

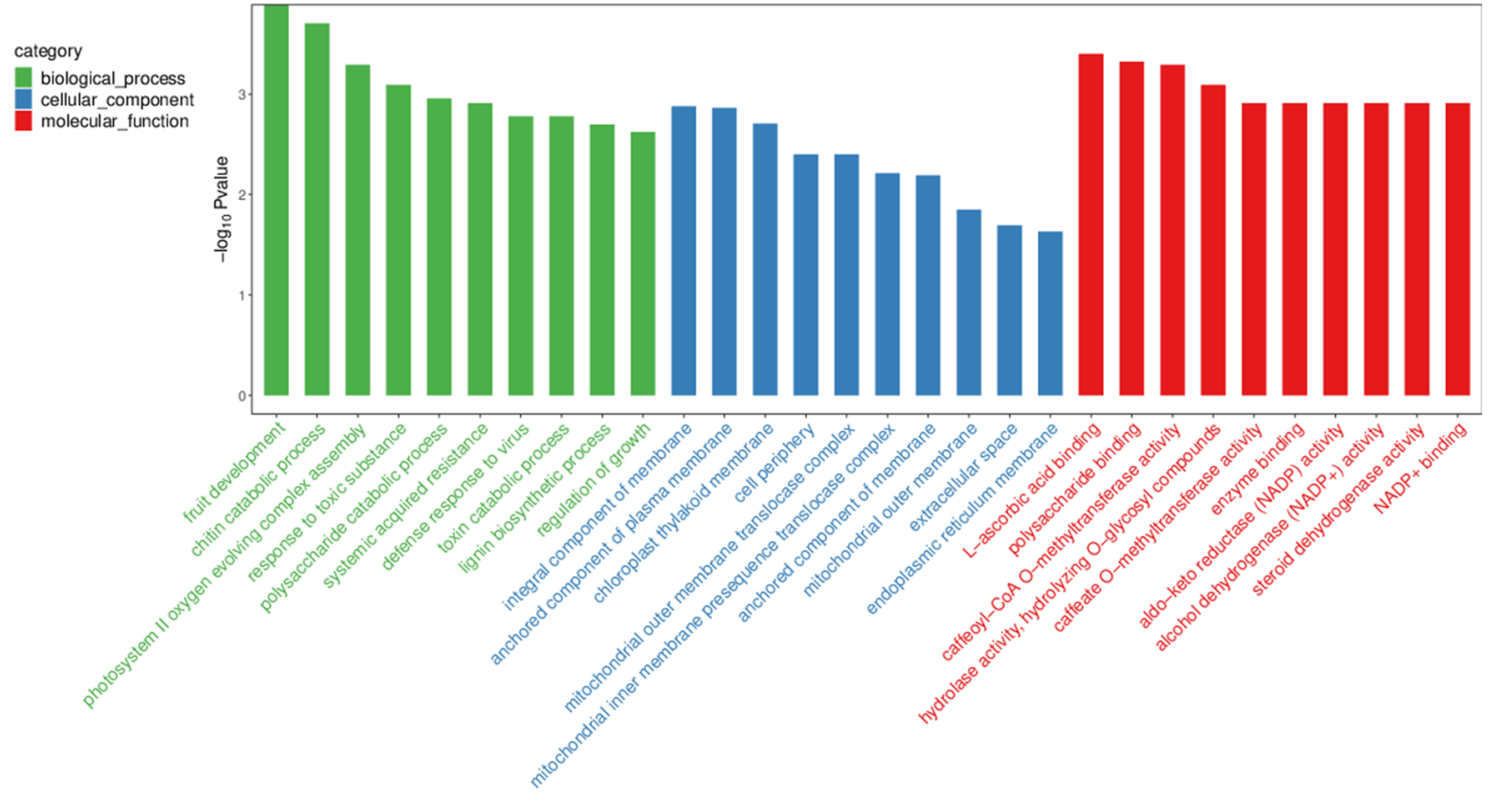

b

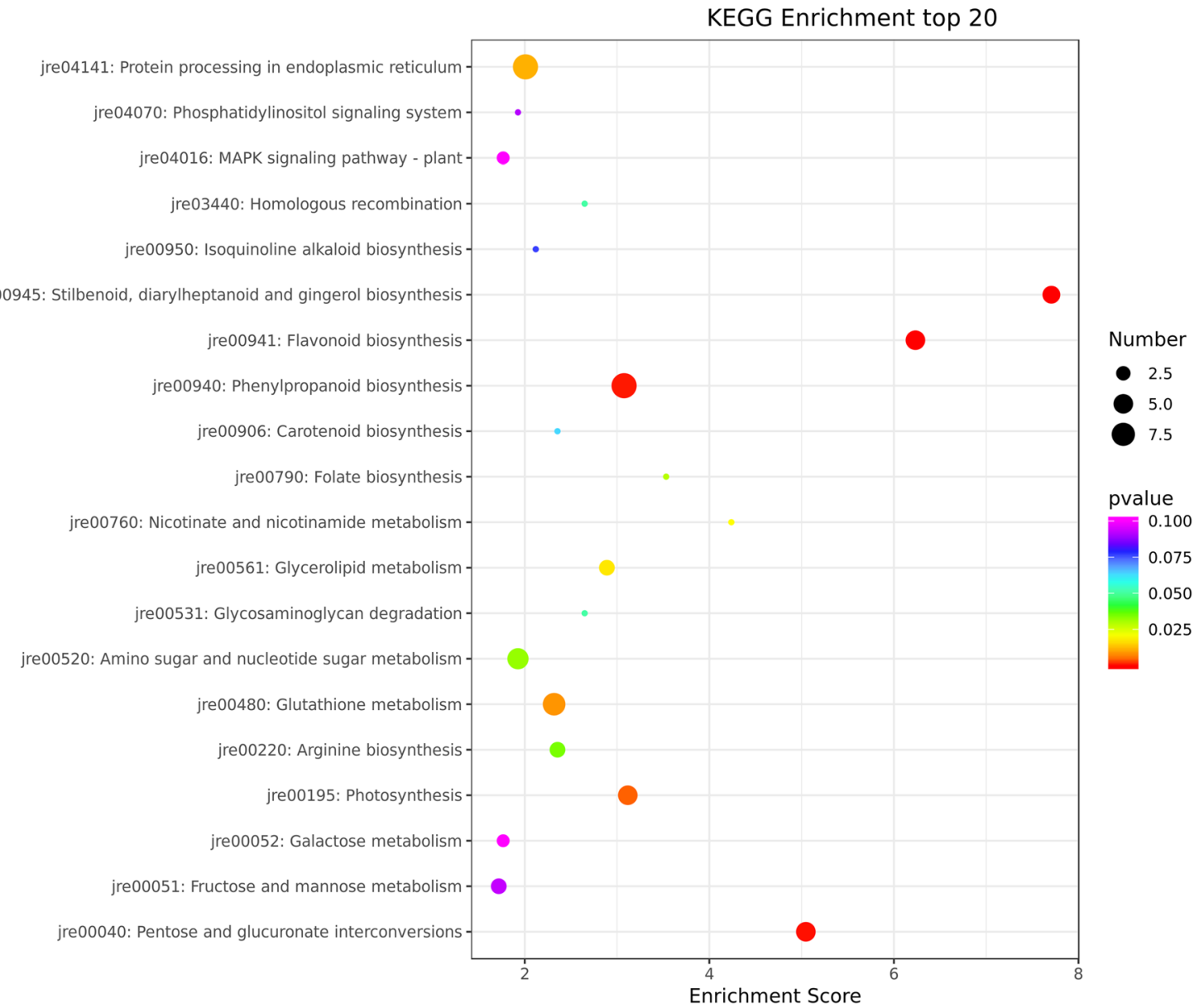

Figure 2. A GO classification of differentially expressed proteins under salt stress. Three main GO categories are summarized: BP, CC, and MF. BP, biological process; CC, cellular component; MF, molecular function. B The top 20 enriched gene ontology (GO) terms in the biological process under $0.6 \% \mathrm{NaCl}$ stress for $48 \mathrm{~h}$. The rich factor indicates the ratio of the number of DEPs in the pathway to the total number of genes in the pathway, and the higher the Rich factor, the higher the degree of enrichment. 


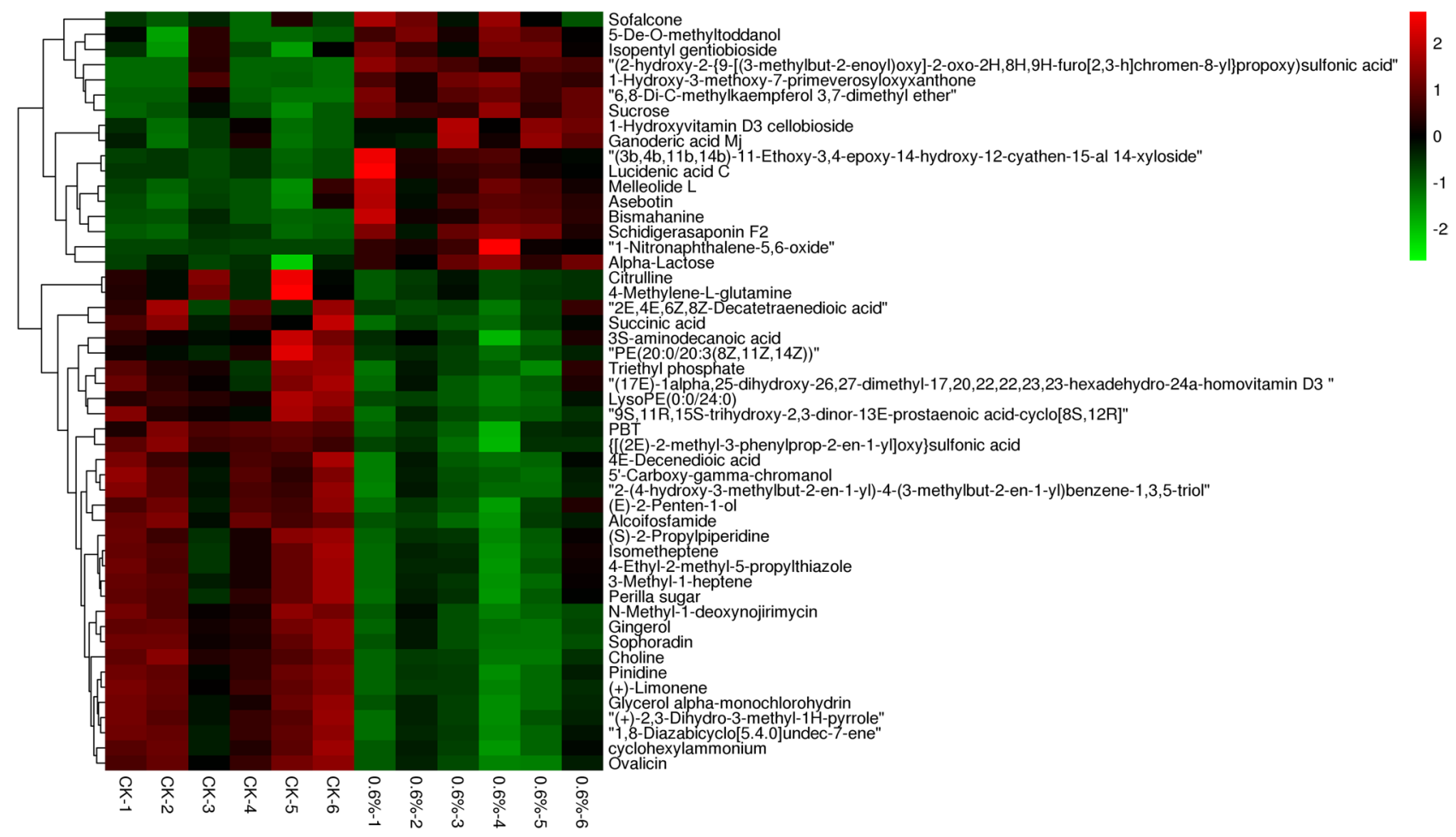

Figure 3. Hierarchical clustering heatmap of different metabolites in pecan leaves after $48 \mathrm{~h}$ of salt stress. The figure was carried out using the OmicShare online analysis cloud platform 6.4 .5 (https://www.omicshare.com/ tools/). "CK" stands for $0 \mathrm{~h}$. "1-6" stands for repetition.

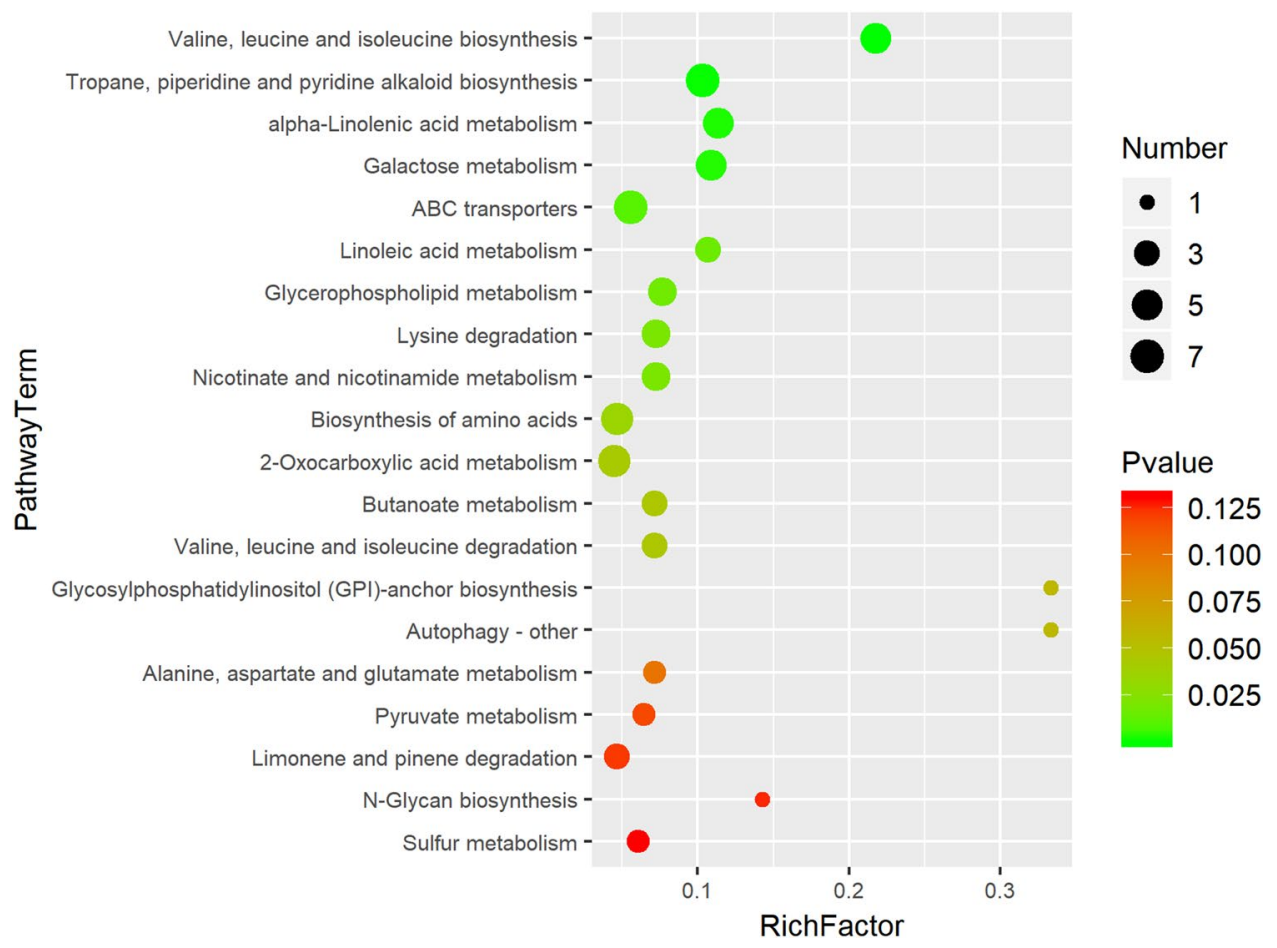

Figure 4. The top 20 enriched $\mathrm{KEGG}$ pathways under $0.6 \% \mathrm{NaCl}$ stress for $48 \mathrm{~h}$. The rich factor indicates the ratio of the number of DEMs in the pathway to the total number of DEMs in the pathway. A higher Rich factor indicates a greater degree of enrichment. 


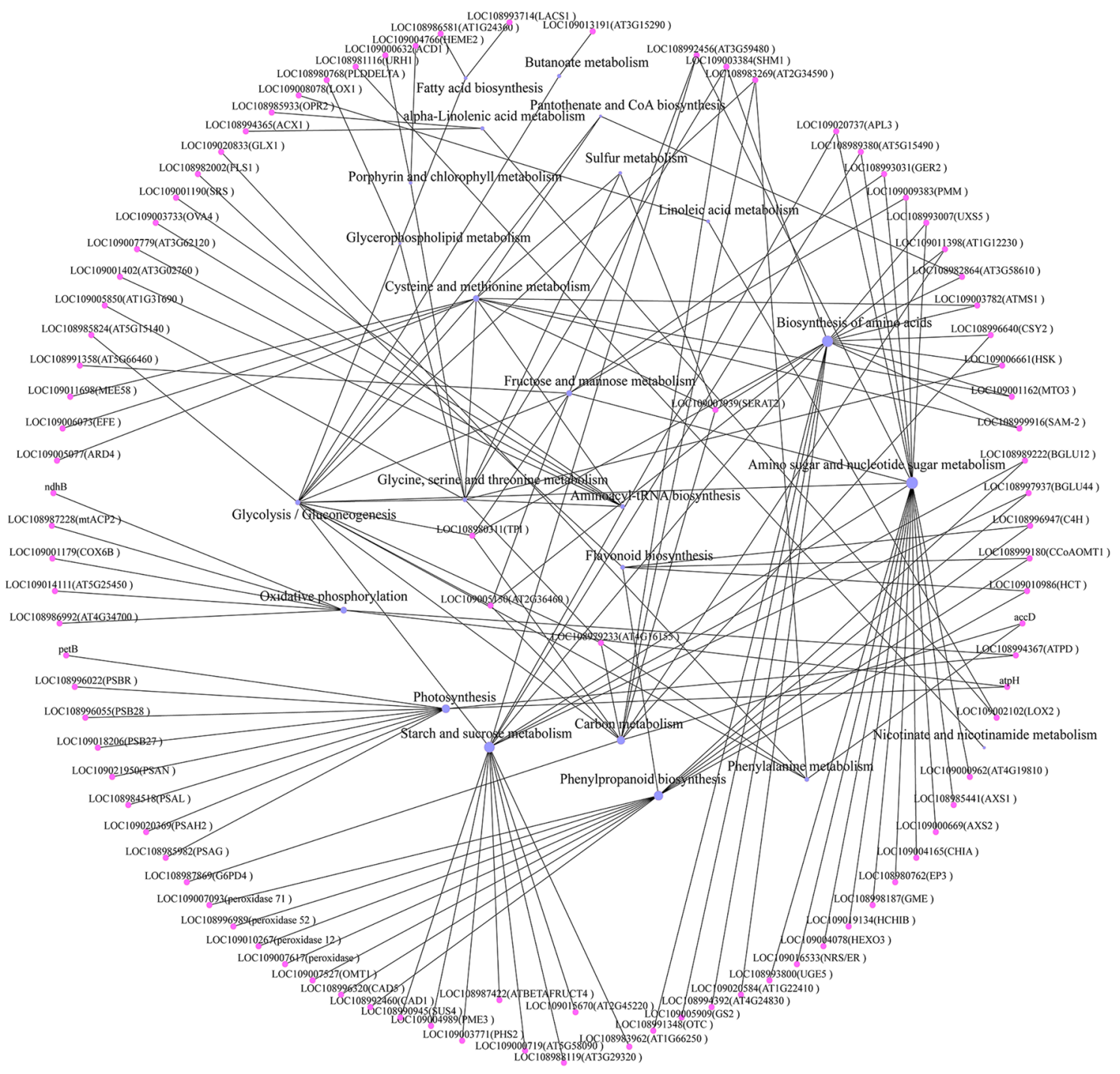

Figure 5. A summary of differentially expressed proteins related to the response of leaves to salt stress and the KEGG pathways involved. The protein is represented by a pink circle; the KEGG pathway it participates in is represented by a blue circle. The size of the blue circle represents the number of proteins involved in its pathway.

\section{Discussion}

Various studies have shown that salt stress has a significant impact on both the photosynthetic structure and efficiency of plant leaves ${ }^{29,30}$. It has been found that the maximum quantum yield of Photosystem II (PSII; Fv/ $\mathrm{Fm}$ ) in salt-sensitive plants is significantly reduced during high salt stress ${ }^{28}$. For example, in the salt-sensitive sweet sorghum, salt stress was also found to significantly reduce $\mathrm{Pn}, \mathrm{Ci}$, and $\mathrm{Gs}^{31}$. In contrast, the Fv/Fm ratio in salt-tolerant rice was essentially unaffected by salt stress ${ }^{32}$, a finding similar to the results of this study (Fig. 1), and confirming that pecans have a high degree of salt tolerance, the Fv/Fm ratio is one of the iconic indicators of salt-tolerant plants. In addition, it has been reported that down-regulation of expression of the photosystem II $10 \mathrm{kDa}$ protein (PSBR) alleviates light-induced non-photochemical quenching (NPQ) and the decline in Fv/ $\mathrm{Fm}^{33}$. Since the PSBR plays an important role in maintaining the stability of the PSII reaction center, including repairing damage to the system, to reduce salt-alkali stress. Then, two PSII repair proteins (PSB27-H1 and PSB27) were identified in this study (Table S1), and the small changes observed in Pn, NPQ, and Fv/Fm may be due to the result of the down-regulation of these proteins. In addition, the data also showed the down-regulation of photosystem I (PSI) reaction center subunits (XI, V, VI, and N) proteins (Table S1). We believe that these may be PSI-protective proteins, with functions similar to that of the PSII repair protein, although this requires verification. Unfortunately, we did not identify DEMs reacting with these photosynthesis-related proteins in the result of the correlation analysis, suggesting that the proteins may play indirect roles in the regulation of the salt-stress response.

As is known, sugar plays a key role in plant stress perception and signal transduction, and can also mediate osmotic regulation and carbohydrate distribution ${ }^{34}$. Consistent with a previous report ${ }^{35}$, this study also identified changes in the levels of various soluble sugars, including sucrose, fructose, and glucose. Twenty DEPs that 


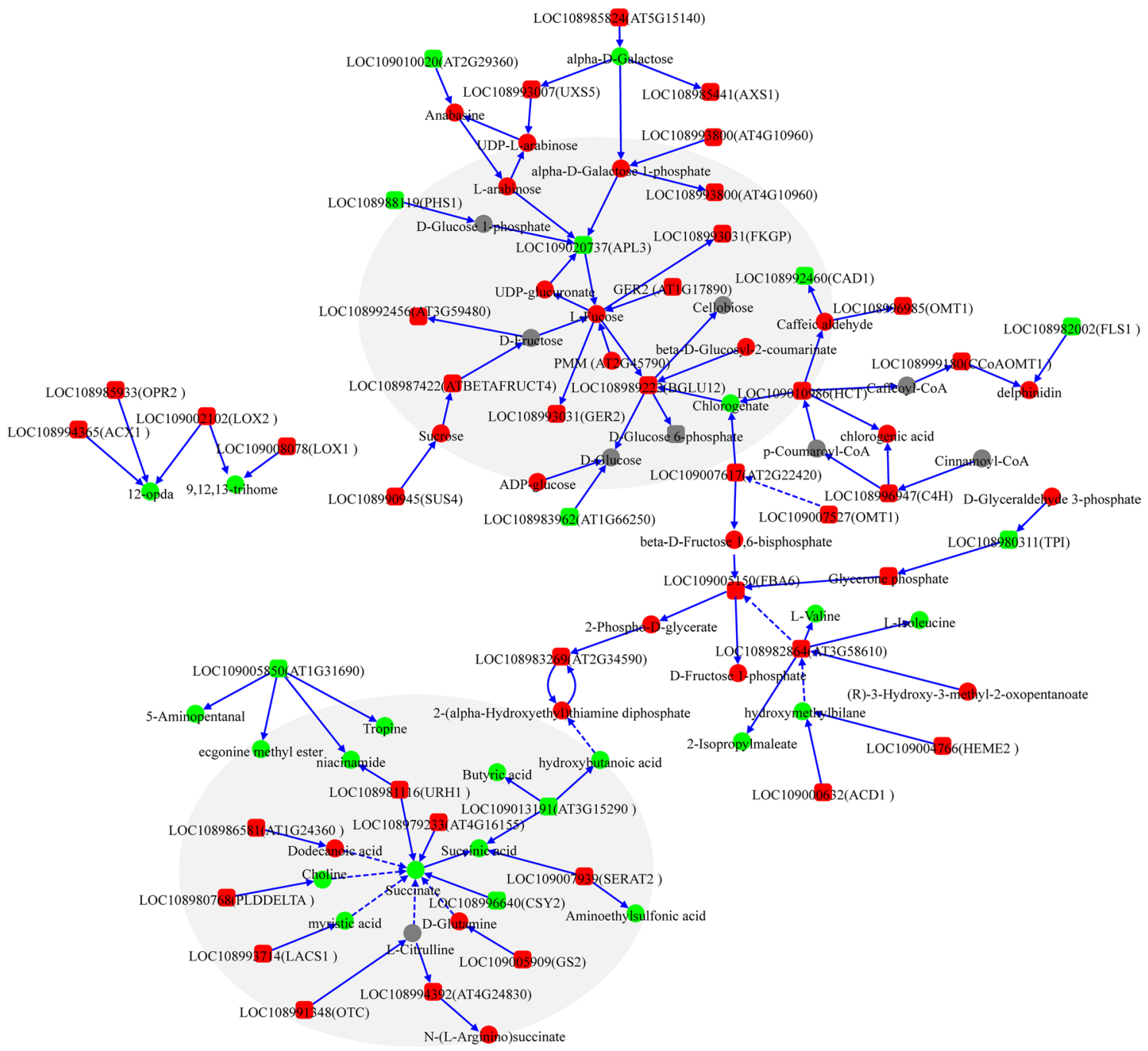

Figure 6. The core biological response pathway of pecan to salt stress. Based on the KEGG pathway, the DEM and DEP correlation analysis results are mapped to a comprehensive metabolic regulation network diagram. The DEMs and proteins are marked as circles and round rectangles, respectively; red indicates upregulation; green indicates downregulation. The key DEPs and DEMs are shown in the light gray background areas.

interacted directly with these sugars were identified, with most of these proteins up-regulated under salt-stress conditions (Fig. 6). This led to the identification of two key metabolites, namely L-fucose and succinic acid. Of these, L-fucose plays an important role in the response of plants to salt stress, and as a defense strategy for protein glycosylation, it participates in regulating the formation of physical barriers (keratin) and activating the immune system, stomata defense, and peptide synthesis ${ }^{36,37}$. Our findings confirm these results. The large increase in L-fucose accumulation $\left(\log _{2}\right.$ Fold change $\left.=2.74\right)$ suggests the immune response may be activated under conditions of salt stress. L-fucose, found to be one of the key nodes in the regulatory network of DEPs and DEMs under salt stress, is known to interact with many proteins, including PMM, FKGP, GER2, and APL3, although its precise function is not understood. In contrast, the succinate-interacting protein CSY2, one of the key nodes in the regulatory network, was found to be down-regulated, possibly due to changes in the tricarboxylic acid cycle (TCA cycle) and mitochondrial respiration. CSY2 may thus assist the adaptation to salt stress in the pecan. This is consistent with previous research reports ${ }^{38}$. It is worth noting that both beta-D-fructose 1,6-bisphosphate $\left(\log _{2}\right.$ Fold change $\left.=2.74\right)$ and its interaction partner fructose 1,6-bisphosphate aldolase (FBA) 6 were found to be up-regulated $\left(\log _{2}\right.$ Fold change $\left.=0.35\right)$. Since the FBA is an important enzyme in the glycolysis/gluconeogenesis pathway, and is located in the chloroplast where it increases carbon flux through the Calvin cycle and assists in stress tolerance by alleviating the inhibition of photosynthesis induced by salt stress ${ }^{39}$. This may be the reason why we did not observe a significant reduction in the photosynthetic rate $(P \mathrm{n})$ after $48 \mathrm{~h}$. This is consistent with previous findings ${ }^{40-42}$. We also observed protein enrichment in the phenylpropane synthesis pathway (BGLU12, HCT, and OMT1) together with an unexpected increase in the accumulation of beta-D-Glucosyl-2-coumarinate and cis-beta-D-Glucosyl-2-hydroxycinnamate, suggesting that the plants were actively responding to salt stress and repairing oxidative damage. However, the chlorogenate showed down-regulation (Table 1), it is clearly the result of salt stress, the precise function requires clarification. Similarly, this study also found that proteins and 
Tropane, piperidine and pyridine alkaloid biosynthesis

\begin{tabular}{|l|l|c|}
\hline \multirow{4}{*}{ Protein } & LOC109005850/primary amine oxidase-like & -0.25 \\
\cline { 2 - 4 } Metabolite & LOC109010020/tropinone reductase homolog & -0.27 \\
& 5-Aminopentanal & -0.37 \\
\cline { 2 - 4 } & Anabasine & 1.96 \\
\cline { 2 - 3 } & Ecgonine methyl ester & -0.98 \\
\cline { 2 - 3 } & Tropine & -0.87
\end{tabular}

Amino sugar and nucleotide sugar metabolism

Protein

LOC109020737/glucose-1-phosphate adenylyltransferase large subunit 1-like isoform X1

LOC108993031/GDP-L-fucose synthase 1-like

LOC108985441/UDP-D-xylose synthase 2-like

LOC108993007/ UDP-XYL synthase 5

LOC108993800/ UDP-D-glucose/UDP-D-galactose

4-epimerase 5

Metabolite

Alpha-D-Galactose 1-phosphate

L-arabinose

UDP-L-arabinose

\begin{tabular}{|l|l|}
\hline-0.91 \\
\hline 0.44 \\
\hline 0.34 \\
\hline 0.40 \\
\hline 1.40 \\
\hline 2.44 \\
\hline 3.96 \\
\hline 0.74 \\
\hline
\end{tabular}

Starch and sucrose metabolism

LOC108983962/ O-Glycosyl hydrolases family 17 protein $\quad-0.46$

LOC108989222/beta-glucosidase 12-like

LOC108990945/sucrose synthase-like

LOC108988119/alpha-1,4 glucan phosphorylase L

Protein

isozyme, chloroplastic/amyloplastic

LOC108987422/acid beta-fructofuranosidase 1, vacuolar

like

LOC109020737/glucose-1-phosphate adenylyltransferase large subunit 1-like isoform X1

Metabolite

Sucrose

\begin{tabular}{|r|r|}
-0.46 \\
\hline 0.47 \\
\hline-0.66 \\
\hline 0.28 \\
\hline-0.91 \\
\hline 2.84 \\
\hline
\end{tabular}

Phenylpropanoid biosynthesis

\begin{tabular}{|c|c|c|}
\hline \multirow{6}{*}{ Protein } & LOC108989222/beta-glucosidase 12-like & 0.47 \\
\hline & $\begin{array}{l}\text { LOC109010986/shikimate O-hydroxycinnamoyltrans- } \\
\text { ferase-like }\end{array}$ & 0.34 \\
\hline & LOC109007617/peroxidase 17-like & 0.27 \\
\hline & LOC108996985/caffeic acid 3-O-methyltransferase & 0.39 \\
\hline & $\begin{array}{l}\text { LOC108992460/probable cinnamyl alcohol dehydroge- } \\
\text { nase } 1\end{array}$ & -0.54 \\
\hline & LOC109007527/caffeic acid 3-O-methyltransferase & 0.49 \\
\hline \multirow{3}{*}{ Metabolite } & Chlorogenate & -1.95 \\
\hline & Beta-D-Glucosyl-2-coumarinate & 2.70 \\
\hline & Cis-beta-D-Glucosyl-2-hydroxycinnamate & 3.01 \\
\hline \multicolumn{3}{|c|}{ Fructose and mannose metabolism } \\
\hline \multirow{3}{*}{ Protein } & LOC108980311/ Triose phosphate isomerase & -0.31 \\
\hline & LOC108993031/GDP-L-fucose synthase 1-like & 0.44 \\
\hline & $\begin{array}{l}\text { LOC109005150/fructose-bisphosphate aldolase 6, } \\
\text { cytosolic }\end{array}$ & 0.35 \\
\hline Metabolite & L-Fucose & 2.74 \\
\hline \multicolumn{3}{|c|}{ Stilbenoid, diarylheptanoid and gingerol biosynthesis } \\
\hline \multirow{3}{*}{ Protein } & LOC108996947/trans-cinnamate 4-monooxygenase-like & 0.34 \\
\hline & LOC108999180/caffeoyl-CoA O-methyltransferase 5 & 0.71 \\
\hline & $\begin{array}{l}\text { LOC109010986/shikimate O-hydroxycinnamoyltrans- } \\
\text { ferase-like }\end{array}$ & 0.34 \\
\hline Metabolite & Chlorogenic acid & 1.19 \\
\hline \multicolumn{3}{|c|}{ Arginine biosynthesis } \\
\hline \multirow{3}{*}{ Protein } & $\begin{array}{l}\text { LOC108991348/ornithine carbamoyltransferase, chlo- } \\
\text { roplastic }\end{array}$ & 0.26 \\
\hline & $\begin{array}{l}\text { LOC108994392/argininosuccinate synthase, chloroplas- } \\
\text { tic-like }\end{array}$ & 0.30 \\
\hline & $\begin{array}{l}\text { LOC109005909/glutamine synthetase leaf isozyme, } \\
\text { chloroplastic-like }\end{array}$ & 0.37 \\
\hline
\end{tabular}




\begin{tabular}{|c|c|c|}
\hline \multicolumn{2}{|l|}{ Pathway } & \multirow{2}{*}{$\frac{\log _{2} \text { (Fold change) }}{1.97}$} \\
\hline \multirow{2}{*}{ Metabolite } & $\mathrm{N}$-(L-Arginino)succinate & \\
\hline & D-Glutamine & 0.85 \\
\hline \multicolumn{3}{|c|}{ Galactose metabolism } \\
\hline \multirow{3}{*}{ Protein } & LOC108993800/UDP-glucose 4-epimerase GEPI48 & 1.40 \\
\hline & $\begin{array}{l}\text { LOC108987422/acid beta-fructofuranosidase 1, vacuolar- } \\
\text { like }\end{array}$ & 0.29 \\
\hline & LOC108985824/galactose mutarotase-like & 0.37 \\
\hline \multirow{2}{*}{ Metabolite } & Alpha-D-Galactose 1-phosphate & 2.44 \\
\hline & Alpha-D-Galactose & 0.65 \\
\hline \multicolumn{3}{|c|}{ Citrate cycle (TCA cycle) } \\
\hline \multirow{3}{*}{ Protein } & LOC108996640/citrate synthase, glyoxysomal-like & -0.83 \\
\hline & $\begin{array}{l}\text { LOC108983269/pyruvate dehydrogenase E1 component } \\
\text { subunit beta-3, chloroplastic }\end{array}$ & 0.28 \\
\hline & $\begin{array}{l}\text { LOC108979233/dihydrolipoyl dehydrogenase 2, } \\
\text { chloroplastic-like }\end{array}$ & 1.21 \\
\hline \multirow{2}{*}{ Metabolite } & Succinate & -1.09 \\
\hline & 2-(Alpha-Hydroxyethyl) thiamine diphosphate & 2.14 \\
\hline \multicolumn{3}{|c|}{ Glycolysis or gluconeogenesis } \\
\hline \multirow{2}{*}{ Protein } & $\begin{array}{l}\text { LOC108983269/pyruvate dehydrogenase E1 component } \\
\text { subunit beta-3, chloroplastic }\end{array}$ & 0.28 \\
\hline & $\begin{array}{l}\text { LOC109005150/fructose-bisphosphate aldolase 6, } \\
\text { cytosolic }\end{array}$ & 0.35 \\
\hline Metabolite & 2-Phospho-D-glycerate & 1.88 \\
\hline \multicolumn{3}{|c|}{ Valine, leucine, and isoleucine biosynthesis } \\
\hline Protein & $\begin{array}{l}\text { LOC108982864/ketol-acid reductoisomerase, chloro- } \\
\text { plastic }\end{array}$ & 0.41 \\
\hline \multirow{4}{*}{ Metabolite } & (R)-3-Hydroxy-3-methyl-2-oxopentanoate & 1.30 \\
\hline & L-Isoleucine & -0.78 \\
\hline & L-Valine & -3.04 \\
\hline & 2-Isopropylmaleate & -2.62 \\
\hline \multicolumn{3}{|c|}{ Alpha-Linolenic acid metabolism } \\
\hline \multirow{3}{*}{ Protein } & LOC108994365/peroxisomal acyl-coenzyme A oxidase 1 & 0.43 \\
\hline & $\begin{array}{l}\text { LOC109002102/linoleate 13S-lipoxygenase 2-1, } \\
\text { chloroplastic-like }\end{array}$ & 0.34 \\
\hline & LOC108985933/12-oxophytodienoate reductase 2-like & 0.41 \\
\hline Metabolite & 12-Opda & -0.96 \\
\hline \multicolumn{3}{|c|}{ Linoleic acid metabolism } \\
\hline \multirow[b]{2}{*}{ Protein } & LOC109008078/probable linoleate 9S-lipoxygenase 5 & 0.53 \\
\hline & $\begin{array}{l}\text { LOC109002102/linoleate 13S-lipoxygenase 2-1, } \\
\text { chloroplastic-like }\end{array}$ & 0.34 \\
\hline Metabolite & 9,12,13-Trihome & -0.79 \\
\hline \multicolumn{3}{|c|}{ Glycerophospholipid metabolism } \\
\hline Protein & LOC108980768/phospholipase D delta-like & 0.46 \\
\hline Metabolite & Choline & -0.65 \\
\hline \multicolumn{3}{|c|}{ Nicotinate and nicotinamide metabolism } \\
\hline Protein & LOC108981116/uridine nucleosidase 1 & 0.36 \\
\hline Metabolite & Niacinamide & -0.75 \\
\hline \multicolumn{3}{|c|}{ Porphyrin and chlorophyll metabolism } \\
\hline \multirow[t]{2}{*}{ Protein } & $\begin{array}{l}\text { LOC109000632/pheophorbide a oxygenase, chloroplas- } \\
\text { tic-like }\end{array}$ & 0.27 \\
\hline & LOC109004766/uroporphyrinogen decarboxylase-like & 0.30 \\
\hline Metabolite & Hydroxymethylbilane & -0.77 \\
\hline \multicolumn{3}{|c|}{ Fatty acid biosynthesis } \\
\hline Protein & $\begin{array}{l}\text { LOC108986581/3-oxoacyl-[acyl-carrier-protein] reduc- } \\
\text { tase } 4 \text { isoform X1 }\end{array}$ & 0.31 \\
\hline & LOC108993714/long chain acyl-CoA synthetase 1-like & 0.42 \\
\hline Metabolite & Dodecanoic acid & 0.88 \\
\hline Metabolite & Myristic acid & -0.84 \\
\hline Sulfur meta & & \\
\hline Continued & & \\
\hline
\end{tabular}




\begin{tabular}{|c|c|c|}
\hline \multicolumn{2}{|l|}{ Pathway } & \multirow{2}{*}{$\begin{array}{c}\log _{2} \text { (Fold change) } \\
0.42\end{array}$} \\
\hline Protein & $\begin{array}{l}\text { LOC109007939/serine acetyltransferase 1, chloroplastic- } \\
\text { like }\end{array}$ & \\
\hline \multirow{2}{*}{ Metabolite } & Aminoethylsulfonic acid & 1.79 \\
\hline & Succinic acid & -0.88 \\
\hline \multicolumn{3}{|c|}{ Butanoate metabolism } \\
\hline Protein & $\begin{array}{l}\text { LOC109013191/uncharacterized protein LOC109013191 } \\
\text { isoform X3 }\end{array}$ & -0.30 \\
\hline \multirow{3}{*}{ Metabolite } & Hydroxybutanoic acid & 0.85 \\
\hline & Butyric acid & -0.72 \\
\hline & Succinic acid & -0.88 \\
\hline \multicolumn{3}{|c|}{ Pantothenate and CoA biosynthesis } \\
\hline Protein & $\begin{array}{l}\text { LOC108982864/ketol-acid reductoisomerase, chloro- } \\
\text { plastic }\end{array}$ & 0.41 \\
\hline Metabolite & L-Valine & 3.04 \\
\hline \multicolumn{3}{|c|}{ Flavonoid biosynthesis } \\
\hline \multirow{4}{*}{ Protein } & LOC108996947/trans-cinnamate 4-monooxygenase-like & 0.34 \\
\hline & LOC108999180/caffeoyl-CoA O-methyltransferase 5 & 0.71 \\
\hline & $\begin{array}{l}\text { LOC108982002/flavonol synthase/flavanone 3-hydrox- } \\
\text { ylase-like }\end{array}$ & -0.28 \\
\hline & $\begin{array}{l}\text { LOC109010986/shikimate O-hydroxycinnamoyltrans- } \\
\text { ferase-like }\end{array}$ & 0.34 \\
\hline \multirow{2}{*}{ Metabolite } & Chlorogenic acid & 1.19 \\
\hline & Delphinidin & 2.52 \\
\hline
\end{tabular}

Table 1. The core KEGG biological pathway and its changes in the correlation analysis results of DEP and DEM in pecan after $48 \mathrm{~h}$ of salt stress.

\begin{tabular}{|l|l|l|l|l|c|}
\hline No & UniProt accession & Gene $^{*}$ & Protein symbol & Unique peptide & Log $_{2}$ (Fold change) \\
\hline 1 & A0A2I4DL15 & AT3G09640 & LOC108981203 & HPDELAHEANNGLDIAVR & -0.46 \\
\hline 2 & A0A2I4E5N6 & AT4G17030 & LOC108986524 & TVNDGSVTGVSR & 0.15 \\
\hline 3 & A0A2I4E7J1 & AT2G21250 & LOC108986997 & TVAQIVLR & -0.56 \\
\hline 4 & A0A2I4E8K1 & AT4G02340 & LOC108987294 & ALAPDLR & -0.24 \\
\hline 5 & A0A2I4EKZ7 & AT4G27670 & LOC108990523 & DGVLYITIPK & 4.05 \\
\hline 6 & A0A2I4EYA1 & AT4G10960 & LOC108993800 & LAGDFGDNLSFHQVDIR & 1.54 \\
\hline 7 & A0A2I4F2X6 & AT3G23600 & LOC108995000 & QFEEVLTAR & -0.14 \\
\hline 8 & A0A2I4F5P0 & AT1G08550 & LOC108995788 & IQTPDGGFFTR & -0.19 \\
\hline 9 & A0A2I4FFZ4 & AT2G29500 & LOC108998475 & ENSAFANTR & 0.34 \\
\hline 10 & A0A2I4FXX6 & AT5G13510 & LOC109003002 & AEIYAQLLGSLK & 0.44 \\
\hline 11 & A0A2I4G6G4 & AT2G36460 & LOC109005150 & YQDELIANAAYIGTPGK & -0.17 \\
\hline 12 & A0A2I4G9H4 & AT5G35630 & LOC109005909 & SILNLSLR & 0.09 \\
\hline 13 & A0A2I4GH76 & AT3G16240 & LOC109007845 & FDDSFSLGSLK & 0.13 \\
\hline 14 & A0A2I4GRR4 & AT1G71695 & LOC109010267 & FVDFMTK & -0.25 \\
\hline 15 & A0A2I4GSI9 & AT3G06035 & LOC109010467 & YTGAGIGSEK & -0.15 \\
\hline 16 & A0A2I4GSU9 & AT1G64770 & LOC109010561 & YETLDQGR & -0.25 \\
\hline 17 & A0A2I4GW66 & AT1G12230 & LOC109011398 & LAYDTHGIIR & -0.38 \\
\hline 18 & A0A2I4GWV0 & AT2G27290 & LOC109011575 & VGISTNETGEK & -0.36 \\
\hline 19 & A0A2I4H153 & AT4G13010 & LOC109012612 & AVQYNAYGGGPDGLQHVEVPVPTPNKDEVLLR & -0.15 \\
\hline 20 & A0A2I4HQ33 & AT5G48480 & LOC109020284 & ASDAIQFYK & 0.26 \\
\hline & & & & \\
\hline
\end{tabular}

Table 2. PRM verification of DEPs in pecans after $48 \mathrm{~h}$ salt stress compared with controls. ${ }^{\star}$ Gene code was derived from Arabidopsis thaliana (TAIR10) (https://www.arabidopsis.org/index.jsp). PRM, parallel reaction monitoring; DEPs, differentially expressed proteins. The related descriptions of these DEPs were shown in Table S1. 
metabolites involved in the biosynthetic pathway of flavonoids were also enriched (Figs. 2B, 5), including the up-regulated C4H, HCT, CCoAOMT1, chlorogenic acid, and delphinium, consistent with previous reports ${ }^{43-45}$. This indicates that the flavonoid biosynthetic pathway in plants is positively regulated under salt stress. In addition, the secondary metabolites of polyphenols, chlorogenic acid and delphinium are known antioxidants and may participate in the response to the adverse effects of salt stress through plant osmotic adjustment. Finally, the protein and metabolite regulatory networks appear to be independent, and the $\alpha$-linolenic acid and linoleic acid metabolic pathways were not found to be related to other metabolic pathways (Fig. 6). Among them, 12-OPDA is an oxidation product of a-linolenic acid, and as a precursor of jasmonic acid that is an important plant hormone. It has an important regulatory role in the plant stress response $e^{46,47}$, and will serve as a key research topic in the future.

Briefly, most of the current research results on the molecular mechanism of salt stress have been undertaken in model plants and food crops, and this is the first comprehensive study to incorporate plant physiology, proteins, and metabolites in an investigation of the responses to salt stress in pecan. The study also provides a theoretical basis for the choice of suitable economically viable trees in high-salt tidal areas. Further functional analysis and genetic translational verification of the key proteins identified is, however, still needed.

\section{Conclusion}

This study analyzed the photosynthetic and fluorescence parameters of pecans under salt stress using iTRAQ and untargeted LC/MS to investigate the response of pecans to salt stress. The results showed the differential expression of 198 DEPs and 538 DEMs after $48 \mathrm{~h}$ of salt stress. Further investigation using protein and metabolomics pathway correlation analysis showed enrichment of 21 core pathways and two key nodes of the salt stress regulatory network, L-fucose and succinate. Finally, PRM analysis verified the expression levels of multiple proteins. In summary, this study provides an important theoretical starting point for future coastal saline-alkali cultivation and related research.

Received: 28 July 2021; Accepted: 13 January 2022

Published online: 03 February 2022

\section{References}

1. Katul, G. G., Oren, R., Manzoni, S., Higgins, C. \& Parlange, M. B. Evapotranspiration: A process driving mass transport and energy exchange in the soil-plant-atmosphere-climate system. Rev. Geophys. 50 (2012).

2. Schiestl-Aalto, P. et al. Linking canopy-scale mesophyll conductance and phloem sugar $\delta^{13} \mathrm{C}$ using empirical and modelling approaches. New Phytol. 229, 3141-3155 (2020).

3. Sun, L. et al. Impacts of salt stress on characteristics of photosynthesis and chlorophyll fluorescence of sorghum seedlings. Sci. Agric. Sin. 45, 3265-3272 (2012).

4. Zhang, J. et al. Transcriptomic analysis reveals potential pathways associated with salt resistance in pecan (Carya illinoensis $\mathrm{K}$. Koch). J. Biotechnol. 330, 17-26 (2021).

5. Shen, Q. et al. Ionomic, metabolomic and proteomic analyses reveal molecular mechanisms of root adaption to salt stress in Tibetan wild barley. Plant Physiol. Biochem. 123, 319-330 (2018).

6. Pang, Q. et al. Comparative proteomics of salt tolerance in Arabidopsis thaliana and Thellungiella halophila. J. Proteome Res. 9, 2584-2599 (2010).

7. Zhao, Y., Zhang, F., Mickan, B., Wang, D. \& Wang, W. Physiological, proteomic, and metabolomic analysis provide insights into Bacillus sp.-mediated salt tolerance in wheat. Plant Cell Rep. (2021).

8. Jia, X. M. et al. Integrated physiologic, proteomic, and metabolomic analyses of Malus halliana adaptation to saline-alkali stress. Hortic. Res. 6, 91 (2019).

9. Lee, D. G. et al. Proteomics analysis of salt-induced leaf proteins in two rice germplasms with different salt sensitivity. Can. J. Plant Sci. 91, 337-349 (2011).

10. Zörb, C. et al. The biochemical reaction of maize (Zea mays L.) to salt stress is characterized by a mitigation of symptoms and not by a specific adaptation. Plant Sci. 167, 91-100 (2004).

11. Yu, J. et al. Physiological and proteomic analysis of salinity tolerance in Puccinellia tenuiflora. J. Proteome Res. 10, 3852-3870 (2011).

12. Wang, Y., Stevanato, P., Lv, C., Li, R. \& Geng, G. Comparative physiological and proteomic analysis of two sugar beet genotypes with contrasting salt tolerance. J. Agric. Food Chem. 67, 6056-6073 (2019).

13. Ahmad, F., Singh, A. \& Kamal, A. Osmoprotective role of sugar in mitigating abiotic stress in plants. In Protective Chemical Agents in the Amelioration of Plant Abiotic Stress: Biochemical and Molecular Perspectives, 53-70 (2020).

14. Zhong, Y. et al. TPST is involved in fructose regulation of primary root growth in Arabidopsis thaliana. Plant Mol. Biol. 103, 511-525 (2020).

15. Chen, C. et al. iTRAQ-based proteomic technique provides insights into salt stress responsive proteins in Apocyni Veneti Folium (Apocynum venetum L.). Environ. Exp. Bot. 180, 104247 (2020).

16. Xin, J. et al. AtPFA-DSP3, an atypical dual-specificity protein tyrosine phosphatase, affects salt stress response by modulating MPK3 and MPK6 activity. Plant Cell Environ. 44, 1534-1548 (2021).

17. Zhu, Y. et al. Quantitative proteomic analysis of Malus halliana exposed to salt-alkali mixed stress reveals alterations in energy metabolism and stress regulation. Plant Growth Regul. 90, 205-222 (2020).

18. Lin, F. et al. A leucine-rich repeat receptor-like kinase, OsSTLK, modulates salt tolerance in rice. Plant Sci. 296, 110465 (2020).

19. Song, R. F., Li, T. T. \& Liu, W. C. Jasmonic acid impairs Arabidopsis seedling salt stress tolerance through MYC2-Mediated repression of CAT2 expression. Front. Plant Sci. 12, 730228 (2021).

20. Yan, F. et al. Melatonin improves $\mathrm{K}^{+}$and $\mathrm{Na}^{+}$homeostasis in rice under salt stress by mediated nitric oxide. Ecotoxicol. Environ. Saf. 206, 111358 (2020).

21. Li, Z., Cheng, B., Zeng, W., Zhang, X. \& Peng, Y. Proteomic and metabolomic profilings reveal crucial functions of $\gamma$-aminobutyric acid in regulating ionic, water, and metabolic homeostasis in creeping bentgrass under Salt Stress. J. Proteome Res. 19, 769-780 (2020).

22. Jung, H. H., Kim, J. Y., Lim, J. E. \& Im, Y. H. Cytokine profiling in serum-derived exosomes isolated by different methods. Sci. Rep. 10, 1-11(2020). 
23. Wiśniewski, J. R. Filter-aided sample preparation for proteome analysis. In Microbial Proteomics, 3-10 (Humana Press, New York, 2018).

24. Pan, T. Z., et al. USP7 inhibition induces apoptosis in Glioblastoma by stabilizing ARF4. Res. Square. https://doi.org/10.21203/ rs.3.rs-552140/v1 (2021).

25. Feng, X. K. et al. Transcriptome and metabolome analysis reveals anthocyanin biosynthesis pathway associated with ramie (Boehmeria nivea (L.) Gaud.) leaf color formation. BMC Genomics 22, 1-15 (2021)

26. Tao, S. S. et al. Haemolymph metabolomic differences in silkworms (Bombyx mori L.) under mulberry leaf and two artificial diet rearing methods. Arch. Insect Biochem. 109, e21851 (2021).

27. Kanehisa, M. \& Goto, S. KEGG: Kyoto encyclopedia of genes and genomes. Nucleic Acids Res. 28, 27-30 (2000).

28. Wei, Y. et al. Plasma membrane proteomic analysis by TMT-PRM provides insight into mechanisms of aluminum resistance in tamba black soybean roots tips. Peer J. 8, e9312 (2020).

29. ElSayed, A. I., Rafudeen, M. S., Gomaa, A. M. \& Hasanuzzaman, M. Exogenous melatonin enhances the reactive oxygen species metabolism, antioxidant defense-related gene expression, and photosynthetic capacity of Phaseolus vulgaris L. to confer salt stress tolerance. Physiol. Plantarum 173, 1369-1381 (2021).

30. Faizan, M. et al. Zinc oxide nanoparticles (ZnO-NPs) induce salt tolerance by improving the antioxidant system and photosynthetic machinery in tomato. Plant Physiol. Biochem. 161, 122-130 (2021).

31. Netondo, G. W., Onyango, J. C. \& Beck, E. Sorghum and salinity: II. Gas exchange and chlorophyll fluorescence of sorghum under salt stress. Crop Sci. 44, 806-811 (2004).

32. Yang, Z., Li, J. L., Liu, L. N., Xie, Q. \& Sui, N. Photosynthetic regulation under salt stress and salt-tolerance mechanism of sweet sorghum. Front. Plant Sci. 10, 1722 (2020).

33. Xue, H. et al. PSBR is required for efficient binding of LHCSR3 to photosystem II-light-harvesting supercomplexes in Chlamydomonas reinhardtii. Plant Physiol. 167, 00094 (2015).

34. Saddhe, A. A., Manuka, R. \& Penna, S. Plant sugars: Homeostasis and transport under abiotic stress in plants. Physiol. Plantarum 171, 739-755 (2021).

35. Gao, Y. et al. Comparative proteomic analysis reveals that antioxidant system and soluble sugar metabolism contribute to salt tolerance in Alfalfa (Medicago sativa L.) leaves. J. Proteome Res. 18, 191-203 (2018).

36. Zhang, L., Paasch, B. C., Chen, J., Day, B. \& He, S. Y. An important role of l-fucose biosynthesis and protein fucosylation genes in Arabidopsis immunity. New Phytol. 222, 981-994 (2019).

37. Zhang, X., Tang, H., Du, H., Bao, Z. \& Shi, Q. Sugar metabolic and N-glycosylated profiles unveil the regulatory mechanism of tomato quality under salt stress. Environ. Exp. Bot. 177, 104145 (2020).

38. Che-Othman, M. H., Jacoby, R. P., Millar, A. H. \& Taylor, N. L. Wheat mitochondrial respiration shifts from the tricarboxylic acid cycle to the GABA shunt under salt stress. New Phytol. 225, 1166-1180 (2020).

39. Zeng, Y. et al. A fructose-1,6-biphosphate aldolase gene from Camellia oleifera: molecular characterization and impact on salt stress tolerance. Mol. Breed. 35, 17 (2015).

40. Tang, J. M., Liao, Q. H., Hu, T., Qi, L. W. \& Li, Z. X. Expression of CCoAOMT from Zingiber officinale roscoe under NaCl stress and its regulatory role in 6-gingerol biosynthesis. Russ. J. Plant Physiol. 68, 286-292 (2021).

41. Zhu, Y. et al. Combined transcriptomic and metabolomic analysis reveals the role of phenylpropanoid biosynthesis pathway in the salt tolerance process of Sophora alopecuroides. Int. J. Mol. Sci. 22, 2399 (2021).

42. Cao, B. L., Li, N. \& Xu, K. Crosstalk of phenylpropanoid biosynthesis with hormone signaling in Chinese cabbage is key to counteracting salt stress. Environ. Exp. Bot. 179, 104209 (2020).

43. Chun, H. J. et al. Lignin biosynthesis genes play critical roles in the adaptation of Arabidopsis plants to high-salt stress. Plant Signal. Behav. 14, 1625697 (2019).

44. Chowdhury, E. M., Choi, B. S., Park, S. U., Lim, H. S. \& Bae, H. Transcriptional analysis of hydroxycinnamoyl transferase (HCT) in various tissues of Hibiscus cannabinus in response to abiotic stress conditions. Plant Omics 5, 305 (2012).

45. Gengmao, Z., Shihui, L., Xing, S., Yizhou, W. \& Zipan, C. The role of silicon in physiology of the medicinal plant (Lonicera japonica L.) under salt stress. Sci. Rep. 5, 1-11 (2015).

46. Löwe, J., Dietz, K. J. \& Gröger, H. From a biosynthetic pathway toward a biocatalytic process and chemocatalytic modifications: three-step enzymatic cascade to the plant metabolite cis-(+)-12-OPDA and metathesis-derived products. Adv. Sci. 7, 1902973 (2020).

47. Maynard, D. et al. One-pot synthesis of bioactive cyclopentenones from a-linolenic acid and docosahexaenoic acid. Biorg. Med. Chem. 26, 1356-1364 (2018).

\section{Acknowledgements}

Ningbo Academy of Agricultural Science funded this research (Grant No. NK201901).

\section{Author contributions}

Y.J. contributed thesis methodology for the experiments and wrote the paper. J.H. performed the data analysis and draft preparation. C.D. carried out supervisory tasks and data verification.

\section{Competing interests}

The authors declare no competing interests.

Additional information

Supplementary Information The online version contains supplementary material available at https://doi.org/ 10.1038/s41598-022-05866-9.

Correspondence and requests for materials should be addressed to J.Z.

Reprints and permissions information is available at www.nature.com/reprints.

Publisher's note Springer Nature remains neutral with regard to jurisdictional claims in published maps and institutional affiliations. 
(c) (i) Open Access This article is licensed under a Creative Commons Attribution 4.0 International cc) License, which permits use, sharing, adaptation, distribution and reproduction in any medium or format, as long as you give appropriate credit to the original author(s) and the source, provide a link to the Creative Commons licence, and indicate if changes were made. The images or other third party material in this article are included in the article's Creative Commons licence, unless indicated otherwise in a credit line to the material. If material is not included in the article's Creative Commons licence and your intended use is not permitted by statutory regulation or exceeds the permitted use, you will need to obtain permission directly from the copyright holder. To view a copy of this licence, visit http://creativecommons.org/licenses/by/4.0/.

(C) The Author(s) 2022 